\title{
Ideal Surgery Time in COVID-19 Process: A Case Report of Pulmonary Hydatid Cyst in Covid Patient
}

\author{
Mehmet Çetin ${ }^{1}$ (D)
}

1 Ömer Halisdemir University Training and Research Hospital, Department of Thoracic Surgery, Niğde, Turkey,

\begin{abstract}
In order to reduce the risk of postoperative mortality and pulmonary/thromboembolic complications during the COVID process, surgery is recommended 4-7 weeks after the diagnosis of COVID in elective cases. We are sharing a case of a patient with a parasitic disease such as hydatid cyst, which was detected incidentally with the diagnosis of COVID-19, who was operated on eight weeks after the diagnosis of COVID-19 and experienced postoperative pulmonary complications.
\end{abstract}

Keywords: Acute Lung Injury, COVID, Hydatid Cyst, Postoperative Complication.

\section{INTRODUCTION}

While the COVID-19 pandemic has caused increased morbidity and mortality all over the world, it has also caused serious changes in surgical operating principles because of COVID related complications (1). While millions of elective surgeries are postponed during the peak times of the pandemic, studies are being conducted on the appropriate surgical time for patients with COVID-19 during the period when elective surgery can be performed (2-4).

Hydatid cyst is a parasitic disease in which echinococcus granulosus is the most common causative agent, and the primary treatment is surgery in the lung, which is the second most common organ (5).

We present a case of pulmonary hydatid cyst with postoperative pulmonary complications, diagnosed during the examinations for COVID-19.

\section{CASE REPORT}

Seventeen years old woman patients, who applied to the COVID outpatient clinic with a positive family history of COVID-19 and cough, showed consolidation in the right lower zone with bilateral infiltrates in the chest X-ray (Figure 1A). Due to positive PCR test and active 
complaints, a $10 \times 9 \mathrm{~cm}$ lesion in the right lower lobe with bilateral ground-glass areas on thorax CT taken during hospitalization was thought to be compatible with hydatid cyst (Figure 1B). For the non-ruptured cyst in accordance with surgical principles, right thoracotomy, cystotomy, and capitonage were performed due to the complete regression of infiltrates in the chest X-ray at the 8th week after COVID diagnosis. While there was no pathological finding in the chest X-ray taken on the first postoperative day, increased bilateral infiltrations, increased infection parameters, loss of appetite, and dyspnea occurred on the postoperative second day (Figure 1C). Broad-spectrum antibiotics were started on the patient who had two control PCR tests that resulted negative, no eosinophilia in hemogram and no production in the sputum culture, and widespread infiltration suggestive of acute lung injury was observed in the thorax $\mathrm{CT}$ taken on the 3rd postoperative day (Figure 2A). Then, steroid treatment was added, and the patient, who had dyspnea and decreased infiltration and infection parameters in the follow-up, was discharged on the 8th day in health (Figure 2B). The patient's pathology was confirmed as a hydatid cyst, did not have any active complaints in the 1st week and 1st-month follow-ups after discharge, and no obvious pathology was observed in the chest X-ray (Figure 2C).

Figure 1. A. Chest $X$-ray at the time of COVID diagnosis. B. Thorax CT at the time of diagnosis C. Postoperative 2th day chest $X$-ray

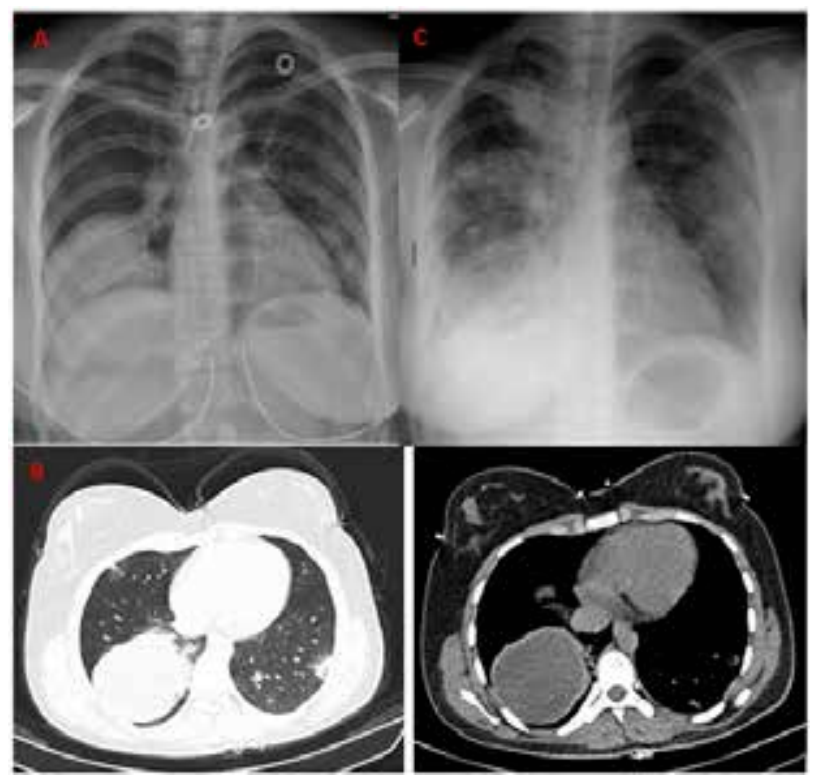

Figure 2. A. Postoperative 4th day thorax CT. B. Discharge chest $\mathrm{X}$-ray. $\mathrm{C}$. Chest $\mathrm{X}$-ray 1 month after discharge

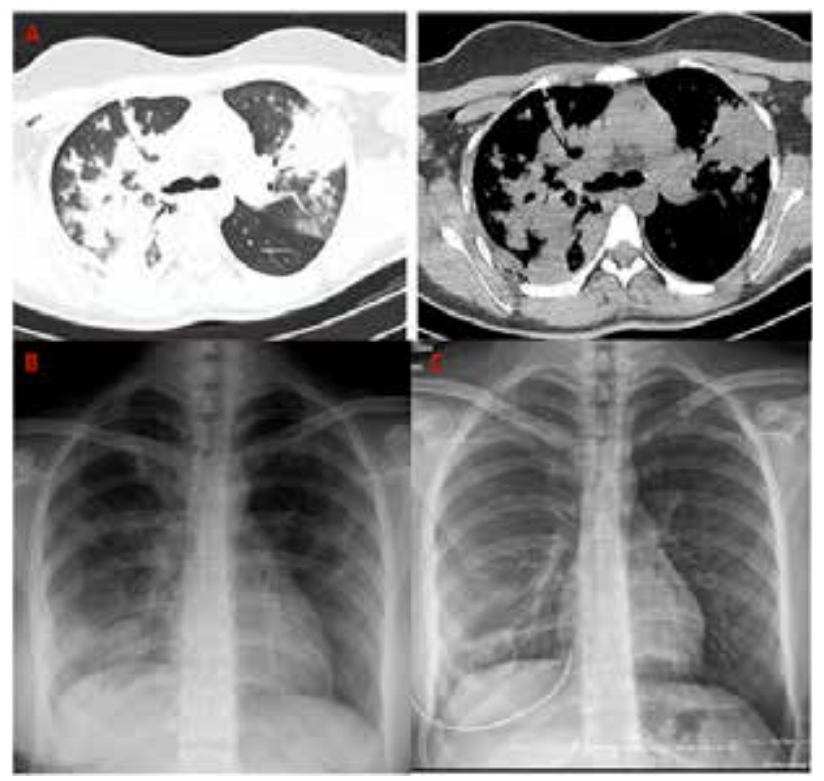

\section{DISCUSSION}

Although there is no possibility to postpone emergency surgeries during the COVID-19 process, efforts have been made to plan elective surgeries together with the pandemic's intensity and establish a guideline to determine the appropriate surgery time, especially in patients with COVID-19. Despite the intensive vaccination programs in developed countries, there are millions of patients who have had COVID-19, and the intense impact of the pandemic still continues due to the low vaccination rates in underdeveloped and developing countries. The question seems to remain on the agenda for a while. (2).

Although there are many studies conducted to decide the time of surgery after COVID, different time periods are mentioned in the results of the study. In a study conducted on 140,000 patients, it was observed that waiting seven weeks from the time of diagnosis in asymptomatic patients and longer waiting times in symptomatic patients reduced the risk of postoperative pulmonary and thromboembolic complications to the same level as in the normal population (2). In addition, there is also a study stating that it is associated with an increased risk in postoperative pulmonary complications and 30-day mortality compared to after surgery to be performed within 4 weeks (3). 
It has been reported that the most common postoperative pulmonary complications are seen after COVID (6). As a matter of fact, in our patient, after waiting 4 weeks after recovery and 8 weeks after diagnosis, respiratory pathology that would cause general deterioration was observed in the surgery, which resulted in a prolonged hospitalization period. Widespread infiltrations in the early postoperative period and rapid steroid response together with thorax CT and clinical findings suggest in favor of pulmonary complications, acute lung injury, especially after COVID.

Hydatid cyst is a parasitic disease in which echinococcus granulosus is usually the causative agent and causes cystic lesions, most commonly in the liver and then in the lung. The basic surgery of hydatid cyst in the lung is cystotomy and capitonage (7). Since it may cause serious complications such as anaphylaxis, hemoptysis, empyema, and pneumonia, especially with the rupture of the cyst, also the possibility of rupture of giant cysts, it is recommended that lung hydatid cysts should be operated as soon as possible after diagnosis (8) As a matter of fact, in a study, at least one ruptured cyst was found in $37 \%$ of lung hydatid cysts, and it was stated that rapid growth was observed in children due to high lung compliance (9). Although there is no recommendation specific to the COVID process, there is an opinion that surgery should be performed as soon as possible in light of the current literature.

In addition to all these, the fact that there are fewer studies in the literature about pediatric patients may also be due to the low rate of diagnosis and testing, although it is evaluated due to the fact that they do not have the disease as severely or asymptomatically as adults (10). However, despite the lack of studies, there are publications stating that elective surgeries with PCR testing can continue with the same complication rates in normal time in studies titled surgery during the COVID-19 process (11). However, since there is no literature on thoracic surgery performed in pediatric patients with COVID-19, it would be useful to consider recommendations for adult patients.

In conclusion, although it is a strong opinion in the literature to wait at least four weeks for elective surgery in patients with a diagnosis of COVID 19 since there are no guidelines yet, it should be evaluated on a case-by-case basis.in diseases such as hydatid cysts.

\section{Declarations}

The author received no financial support for the research and / or authorship of this article. There is no conflict of interest.

\section{REFERENCES}

1. Yadava OP. Post-COVID elective surgery-'to be or not to be'. Indian J Thorac Cardiovasc Surg. 2021;37(3):1-2.

2. COVIDSurg Collaborative, GlobalSurg Collaborative. Timing ofsurgery following SARS-CoV-2 infection: an internationalprospective cohort study. Anaesthesia 2021; 76: 748 - 58.

3. Jonker PKC, van der Plas WY, Steinkamp PJ, Poelstra R, Emous M, van der Meij W, et al. Perioperative SARS-CoV-2 infections increase mortality, pulmonary complications, and thromboembolic events: A Dutch, multicenter, matched-cohort clinical study. Surgery. 2021;169(2):264-274.

4. COVIDSurg Collaborative; GlobalSurg Collaborative. Timing of surgery following SARS-CoV-2 infection: an international prospective cohort study. Anaesthesia. 2021;76(6):748-758.

5. Bagheri R, Haghi SZ, Amini M, Fattahi AS, Noorshafiee S. Pulmonary hydatid cyst: analysis of 1024 cases. Gen Thorac Cardiovasc Surg. 2011;59(2):105-9.

6. COVIDSurg Collaborative. Mortality and pulmonary complications in patients undergoing surgery with perioperative SARS-CoV-2 infection: an international cohort study. Lancet. 2020;396(10243):27-38.

7. Ksia A, Fredj MB, Zouaoui A, Kechiche N, Belhassen S, Mosbahi S, et al. Capitonnage seems better in childhood pulmonary hydatid cyst surgery. J Pediatr Surg. 2020;55(4):752-755

8. Usluer O, Ceylan KC, Kaya S, Sevinc S, Gursoy S. Surgical management of pulmonary hydatid cysts: is size an important prognostic indicator? Tex Heart Inst J. 2010;37(4):429-34.

9. Hamouri S, Odat H, Syaj S, Hecker E, Alrabadi N. Rupture of pulmonary hydatid cyst in pediatrics: A cross-sectional study. Ann Med Surg (Lond). 2021;62:31-36.

10. Mehta NS, Mytton OT, Mullins EWS, Fowler TA, Falconer CL, Murphy OB, et al. SARS-CoV-2 (COVID-19): What Do We Know About Children? A Systematic Review. Clin Infect Dis. 2020;71(9):2469-2479.

11. Okonkwo INC, Howie A, Parry C, Shelton CL, Cobley S, Craig R, et al. The safety of paediatric surgery between COVID-19 surges: an observational study. Anaesthesia. 2020;75(12):1605-1613. 